\title{
Adolescent Idiopathic Scoliosis Progression and Quality of Life. Correlation between SSS-IoT Risk Scale Score and the Main Factors Determining the Disease Potential Progression
}

de Araujo Ono $\mathrm{AH}^{* 1}$, Marcon $\mathrm{RM}^{1}$, Mancuso Filho $\mathrm{JA}^{1}$, Guiroy $\mathrm{A}^{2}$, Cristante $\mathrm{AF}^{1}$ and de Barros Filho $\mathrm{TEP}^{1}$

${ }^{1}$ Faculty of Medicine, University of São Paulo, Hospital das Clínicas, Institute of Orthopedics and Traumatology, São Paulo, Brazil

${ }^{2}$ Spanish Hospital, Mendoza, Argentina

*Corresponding author: de Araujo Ono AH, Faculty of Medicine, University of São Paulo, Hospital das Clínicas, Institute of Orthopedics and Traumatology, São Paulo, Brazil, Tel: +5511982812961, E-mail: allanmedcoluna@gmail.com

Citation: de Araujo Ono AH, Marcon RM, Mancuso Filho JA, Guiroy A, Cristante AF, et al. (2021) Adolescent Idiopathic Scoliosis Progression and Quality of Life. Correlation between SSS-IoT Risk Scale Score and the Main Factors Determining the Disease Potential Progression. J Orthop Physiother 4(1): 105. doi: 10.15744/2639-930X.4.105

Received Date: May 09, 2021 Accepted Date: June 02, 2021 Published Date: June 04, 2021

\begin{abstract}
Introduction: It has been identified several factors that can determine the severity and progression of adolescent idiopathic scoliosis (AIS). The natural evolution of this disease has been extensively studied in different populations. However, no practical tool encompasses the main factors determining this disease's potential progression.
\end{abstract}

Objectives: To correlate the Scoliosis Severity Score (SSS-IoT) scale with the SRS-30 quality of life questionnaire.

Methods: We have conducted a cross-sectional observational study evaluating adolescents with idiopathic scoliosis on the waiting list for surgical procedures. The SRS-30 quality of life questionnaire and the SSS scale was applied. The Cobb values were calculated based on the inclusion day on the waiting list and the survey day. Based on the SSS scale, the patients have grouped in A: less severe scoliosis ( 3 - 8 points); B: mild severe scoliosis ( 9 - 12 points) and C: severe scoliosis (13 - 16 points) and correlated to the quality of life questionnaire and Cobb values (Pearson's test).

Results: Mean age was $15,3 \pm 2,5$ years $\left(86,7 \%\right.$ female). The mean initial Cobb angle was $55.4^{\circ}$ (first day on the waiting list) and $75.5^{\circ}$ (last measurement), resulting in $19.1^{\circ}$ mean progression. Pearson's correlation between SSS and SRS-30 was $-0.318(p$-value $=0,016)$, meaning the higher the progression risk, the lower the quality of life.

Conclusion: In adolescents with idiopathic scoliosis, the SSS scale was directly proportional to the main curve Cobb angle progression's risk. There is a statistical correlation between the SRS-30 quality of life questionnaire and the increased risk of progression correlated to lower quality of life.

Keywords: Adolescent Idiopathic Scoliosis; Risk Scale; Quality of Life; Scoliosis Surgery; Scoliosis Treatment; Waiting List; Orthopaedic Surgery 


\section{Introduction}

According to the Scoliosis Research Society (SRS), scoliosis is a three-dimensional deformity in which the curvature in the frontal view, measured by the Cobb method, is higher than $10^{\circ}$. Adolescent idiopathic scoliosis (AIS) is the most common scoliosis type, affecting youngers between 10 to 18 years old. To date, despite the growing number of genetic and hormonal studies in this field, the main factors that cause AIS are still not well clarified [1].

In adolescents, the prevalence of scoliosis higher than $10^{\circ}$ undertakes $20-30 / 1000$ person and scoliosis greater than $40^{\circ}$ in less than 1/1000 [2]. The higher scoliosis angles are more prevalent in women, reaching 10:1 in curves above 30 grades [3].

The adolescent idiopathic scoliosis's surgical treatment is indicated to growing adolescents with progressive curves greater or equal to 45 degrees or those with skeletally mature and scoliosis higher than 50 degrees. The main treatment objective is to prevent scoliosis progression and reach some degrees of correction. The correction treatment consists of surgery with a posterior approach or an anterior-posterior combined approach with a placed of pedicle screws and nails [1].

The search for surgical treatment for adolescent idiopathic scoliosis has increased worldwide and undergone significant improvements in recent years, mainly due to the implementation of new technologies aimed to reduce the risks inherent to the procedure, such as the use of intraoperative neurophysiology that leads to a reduced risk of neurological injuries and the use of anti-fibrinolytic agents that reduce bleedings and the need for multiple blood transfusions [4-6].

The crescent search for the surgery, the needed for precise instruments, and its high costs lead to the establishment of waiting lists in countries with public health systems. In the same way, in more developed countries like Canada and the United Kingdom, the waiting on the list for this surgery reaches 6 to 12 months [5].

Ahn et al. (2011) have evaluated 216 patients with idiopathic scoliosis and found that the time between the start of the waiting list must not exceed more than three months.

The risk of scoliosis progression in adolescents who wait longer than six months leads to a higher risk of prolonged stays, longer surgical times, lower levels of scoliosis corrections, and a greater risk for a second surgery. Ahn et al. (2011) have evaluated 216 patients with idiopathic scoliosis and found that the time between the start of the waiting list must not exceed more than three months.

A second procedure was needed in 14,8\% of patients waiting longer than six months and 1,6\% for those who waited for a shorter period [6].

Similar results were found by Miyanji et al., showing that patients with a long time on the waiting list are subject to prolonged surgical times, extended length of stays, more bleeding, and a more significant scoliosis progression [7].

Avanzi et al., in a study with 51 patients, have shown that the time on the waiting list for surgical treatment to AIS ranges from 2 to 180 months in a highly specialised hospital in the public health system in Brazil [8].

Carneiro et al. showed an increase of $128 \%$ in the number of patients on the waiting list for surgical treatment to idiopathic scoliosis in 5 years, from 374 in 2007 to 792 in 2009 [9,10].

Currently, in the Clinics Hospital, there are more than 200 patients on the waiting list for scoliosis correction surgery, and most of them have AIS. There is a chronological order to this surgery, and most of the patients suffer from long waiting times to the surgery, sometimes reaching years. 
Ono et al. (2019) have developed one progression risk scale called Scoliosis Severity Score (SSS), based on a systematic review (Figure 1). This scale was built to identify the patients at increased risk for disease progression, reduce the waiting time and optimise the use of resources, reducing the length of stays, the operating rooms use, blood components, and the need for additional procedures and corrections [11].

The SSS-IoT risk scale has eight progressions' criteria based on the literature. The authors' based assigned scores for progression ranged from 0 to 3 related to irrelevant to highly relevant, respectively (Table 1 ).

\begin{tabular}{|c|c|c|}
\hline Clinical/Radiological characteristic & Range & Point \\
\hline \multirow{4}{*}{ Cobb in the beginning } & $<20$ & 0 \\
\hline & $20^{\circ}-30^{\circ}$ & 1 \\
\hline & $>30$ & 2 \\
\hline & $<6^{\circ}$ & 0 \\
\hline \multirow{2}{*}{1 year progression } & $6^{\circ}-10^{\circ}$ & 1 \\
\hline & $>10$ & 2 \\
\hline \multirow{3}{*}{ Risser score } & Iell & 2 \\
\hline & months & 0 \\
\hline & 3-6 months & 1 \\
\hline \multirow{4}{*}{ Waiting time (immature skeleton)* (full mature +1 pt/year) } & $>$ months & 2 \\
\hline & 12 months & 3 \\
\hline & Thoracic principal & 2 \\
\hline & Combined & 2 \\
\hline \multirow{2}{*}{ Type of curve } & Thoracic-lumbar & 1 \\
\hline & Lumbar & 1 \\
\hline \multirow{2}{*}{ Apical vertebra } & Nash Moe $>3$ & 1 \\
\hline & Lenke c & 1 \\
\hline \multirow{3}{*}{ Self-image } & No aesthetic concern & 0 \\
\hline & Moderate aesthetic concern & 1 \\
\hline & High esthetic concern & 2 \\
\hline \multirow{3}{*}{ Thoracic Kyphosis } & Normal & 1 \\
\hline & Low Kyphosis & 2 \\
\hline & Hyper Kyphosis & 1 \\
\hline
\end{tabular}

Table 1: Scoring scale created for risk stratification of patients with adolescent idiopathic scoliosis (SSS - Scoliosis Severity Score)

\section{Objectives}

This present study aims to correlate the Scoliosis Severity Score (SSS) scale with the SRS-30 quality of life questionnaire in adolescents with adolescent idiopathic scoliosis wait in the list for surgical scoliosis correction.

\section{Methods}

A cross-sectional observational study was conducted, including patients with AIS on the waiting list for surgery, carried out in a large, specialised reference centre for adolescent idiopathic scoliosis treatment.

This study included all patients on the waiting list for scoliosis correction surgery and who agreed to participate, non surgical patients are not listed, they are reffered to secondary hospital for conservative treatment. All patients were physically evaluated, and the SSS scale was applied based on each patient's radiological images (Figure 1). Cobb angle were measured, by one single author (AHO) using digital 36" full body xrays, in the software Phillips iSite ${ }^{\circledR}$ 
The patients answered the Scoliosis Research Society (SRS-30) quality-of-life questionnaire, with 30 questions grouped into five domains (function/activity, self-image/appearance, mental health, and satisfaction with handle). Twenty-four questions are to all patients, and the lasts six are to the patients that already have undergone surgery and was not used in this study. It was obtained the mean values and standard deviations of the quality of life of all groups.

This questionnaire's score is inversely correlated to the quality of life in patients with idiopathic scoliosis. The higher the score, the lower the quality of life. This questionnaire was previously translated and validated in Portuguese by Oliveira et al. (2010) [12].

The statistics were conducted using a decision model and regression analysis. The dependent variable for the regression analysis was the quality of life measured by the SRS-30 questionnaire, and the independent variables were all the disease's characteristics.

After the physical and radiological evaluation, the patients were grouped according to the SSS scale score and the SRS-30 questionnaire. Each patient's score on the scale and in the questionnaire was then compared, and the values were correlated using Pearson t-test, presented in scatter plots.

The SSS-IoT scale was then correlated with the measured Cobb angle values and with the scores in the five domain of the SRS-30 qualify-of-life questionnaire. All the values greater than 0,3 was considered correlated.

According to the score in the SSS-IoT scale, the adolescents were grouped into three categories as follow: (A - less severe/aggressive scoliosis - 3 to 8 points), (B - mild severe/aggressive scoliosis - 9 to 12 points) and (C - severe/aggressive scoliosis - 13 to 16 points).

The Institution's Ethics committee approved this research (CAPPesq approval number 780.768). All patients or guardians were informed about the research, signed the free, informed consent term and were allowed to withdraw the research at any time, without interference in their treatment.

\section{Results}

It was contacted 77 patients regularly attended on the waiting list for surgical treatment by phone calls, and 63 agreed to participate and have performed an initial screening. One patient withdrawal for personal reasons and with congenital scoliosis, resulting in 59 patients included in this study.

After the initial evaluation, three patients did not have an adequate radiological image to allow the analysis. Eight were excluded due to the defective or incorrect filling of the SRS-30 questionnaire (more than one option answered or the presence of erasures). After the screening, 48 patients with complete data were included to perform the statistical analysis.

The mean age was 15,29 $\pm 2,47$ years (86,7\% female). The mean initial Cobb values were 55,4 degrees, and the mean Cobb in the final physical examination was 74,54 degrees, meaning a mean increase of 19,12 degrees. The SSS-IoT risk scale has shown a mean score of 11,27 (minimal score of 6 and a maxim score of 16).

Pearson's correlation test between the SSS-IoT risk scale and Cobb angle has shown a moderate correlation of $0,434(p=0,005)$. The logistic regression has shown an increment of 0,047 points in the SSS-IOT scale to each 1 grade in the Cobb angle $(p=0,005)$.

The proposed SSS-IoT risk scale was inversely correlated with the scores in the SRS-30 quality of life questionnaire. Pearson's test has shown a correlation of $-0,318$, meaning that the higher the risk of progression, the lower the quality of life.

The mean score in the SRS-30 quality of life questionnaire was $65,55 \pm 2,23$ (minimal 30 and maxim 107). The adjusted mean and standard deviation scores in this questionnaire to those classified with 1 to 5 points was $2,85 \pm 0,71$, respectively. 
Logistic regression was performed to know the influence of the SSS-IoT risk scale on the patients' quality of life. There is a reduction of 0,053 in the SSS-IoT risk scale to each point in the SRS-30 questionnaire.

The SSS-IoT risk scale showed an inverse association between the patient's age and the risk of scoliosis progression. There is a decrease of 0,438 [ $95 \%$ CI $-0,72$ to $-0,156]$ in the risk of scoliosis progression to each patient's year.

According to scoliosis' severity, 12 patients were grouped in category A (less severe/aggressive scoliosis), 19 patients in category B (mild, severe/aggressive scoliosis) and 17 in category C (severe/aggressive scoliosis).

In the analysis by a group of severity, the patients in group A has shown the highest mean score in the SRS-30 questionnaire (3,29 $\pm 0,39$ ), followed by group C $(2,68 \pm 0,68)$ and group $B(2,65 \pm 0,81), p=0,015$ (Figure 1$)$. The mean score and standard deviation for all patients were $2,86 \pm 0,71$.

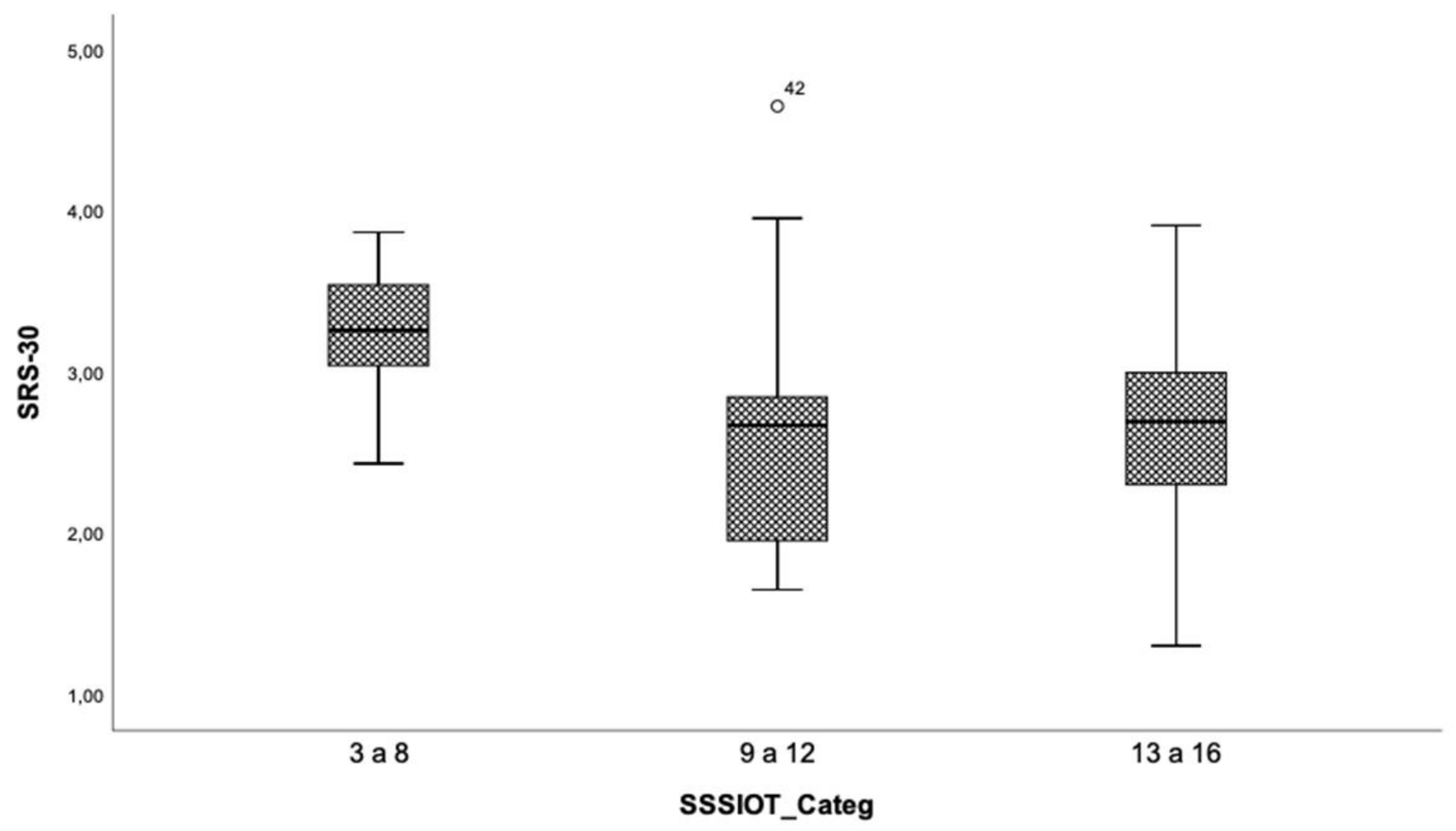

Figure 1: Mean SSS-IoT risk classification scale scores and standard deviation according to the patients classified in group A ( 3 to 8 points), B ( 9 to 12 points) and C (13 to 16 points) by the SRS-30 questionnaire

Each domain in the SRS-30 questionnaire has equality contributed to the mean score of patients. The lowest mean scores were related to the patients' self-image perception $(2,57 \pm 0,88)$, followed by mental health $(2,82 \pm 0,83)$.

When each domain was analysed separately by a group of severity, the adolescents in group A (less severe/aggressive scoliosis) have shown the highest scores in all domains of the quality of life questionnaire, with a higher difference between the function/activity and self-image domains. In the satisfaction with the treatment domain, there was no statistical difference. The results in the qualityof-life questionnaire in all domains are shown in Table 2.

The time on the waiting list was the main factor that has contributed to the SSS-IOT scale (26,9\%), followed by the type of curve $(17,8 \%)$ and initial Cobb angle values (16,9\%). 


\begin{tabular}{|c|c|c|c|c|c|c|c|c|}
\hline \multirow{2}{*}{ Domain } & \multirow{2}{*}{ SSS-IOT Category } & \multirow{2}{*}{$\mathbf{N}$} & \multirow{2}{*}{ Mean } & \multirow{2}{*}{ SD } & \multicolumn{2}{|c|}{ Mean 95\% CI } & \multirow{2}{*}{ Min } & \multirow{2}{*}{$\operatorname{Max}$} \\
\hline & & & & & Lower & Upper & & \\
\hline \multirow{4}{*}{ Function/Activity } & 3 a 8 & 15 & 3,56 & 0,68 & 3,1828 & 3,9372 & 2,00 & 4,60 \\
\hline & 9 a 12 & 16 & 2,78 & 0,91 & 2,2907 & 3,2593 & 1,60 & 5,00 \\
\hline & 13 a 16 & 17 & 2,66 & 0,73 & 2,2849 & 3,0327 & 1,40 & 4,20 \\
\hline & Total & 48 & 2,98 & 0,86 & 2,7292 & 3,2291 & 1,40 & 5,00 \\
\hline \multirow{4}{*}{ Pain } & 3 a 8 & 15 & 3,39 & 0,67 & 3,0158 & 3,7575 & 1,60 & 4,40 \\
\hline & 9 a 12 & 16 & 2,74 & 1,24 & 2,0786 & 3,3964 & 1,20 & 5,00 \\
\hline & 13 a 16 & 17 & 2,79 & 0,89 & 2,3306 & 3,2459 & 1,00 & 4,20 \\
\hline & Total & 48 & 2,96 & 0,99 & 2,6713 & 3,2453 & 1,00 & 5,00 \\
\hline \multirow{4}{*}{ Self-image } & 3 a 8 & 15 & 3,09 & 0,73 & 2,6823 & 3,4955 & 1,50 & 4,17 \\
\hline & 9 a 12 & 16 & 2,26 & 0,80 & 1,8352 & 2,6857 & 1,17 & 4,17 \\
\hline & 13 a 16 & 17 & 2,37 & 0,94 & 1,8901 & 2,8550 & 1,00 & 4,17 \\
\hline & Total & 48 & 2,56 & 0,89 & 2,2999 & 2,8181 & 1,00 & 4,17 \\
\hline \multirow{4}{*}{ Mental Health } & 3 a 8 & 15 & 3,12 & 0,60 & 2,7883 & 3,4517 & 2,00 & 4,20 \\
\hline & 9 a 12 & 16 & 2,65 & 0,98 & 2,1286 & 3,1714 & 1,00 & 4,60 \\
\hline & 13 a 16 & 17 & 2,71 & 0,91 & 2,2373 & 3,1745 & 1,20 & 4,60 \\
\hline & Total & 48 & 2,82 & 0,86 & 2,5671 & 3,0662 & 1,00 & 4,60 \\
\hline \multirow{4}{*}{ Satisfaction } & 3 a 8 & 15 & 3,37 & 0,77 & 2,9420 & 3,7913 & 1,00 & 4,50 \\
\hline & 9 a 12 & 16 & 3,28 & 1,11 & 2,6897 & 3,8728 & 1,00 & 5,00 \\
\hline & 13 a 16 & 17 & 3,32 & 1,21 & 2,7008 & 3,9463 & 1,50 & 5,00 \\
\hline & Total & 48 & 3,32 & 1,03 & 3,0227 & 3,6231 & 1,00 & 5,00 \\
\hline
\end{tabular}

Table 2: Mean distribution by domain in the SRS-30 quality-of-life questionnaire

\section{Discussion}

The waiting lists for surgeries are a common issue in countries with free public health systems, creating a sense of dissatisfaction and political concern due to the prolonged-suffering, decreasing of the pathological condition, pain and, in some cases, increased mortality [13]. The physicians and patients perceive that the waiting lists are not fair, leading to increased dissatisfaction [15].

According to the Brazilian Federal Government System Regulation (SISREG) estimates, more than 800 thousand people were waiting on lists for elective surgery in the public health system in 2017, most of them for orthopaedic surgeries [14].

The ability to analyse the progression by the classification score developed in our study has shown a correlation with the decrease in life quality. This SSS-IoT risk score, developed to meet the demand in our service, has demonstrated a valuable tool to analyse scoliosis progression. Based on the risk of progression, this SSS-IoT enables us to organise the waiting list since the Cobb score increase is directly correlated with its evolution.

Future analysis with the SSS-IoT risk scale will be necessary to predict which factors have higher importance to determine the Cobb angle evolution correctly. In this study, it was also possible to infer that patients with higher scoliosis scores on the SSS-IoT scale have lower scores in the quality-of-life questionnaire. In other words, those patients with moderate to severe progression have shown a lower quality of life in comparison to patients with stable, less severe scoliosis.

Our study has observed that patients with a higher risk of scoliosis progression must prioritise treatment and surgery. When grouped into categories, the patients with 9 to 12 points (moderately, severe scoliosis) and those with 13 to 16 points (severe/ aggressive scoliosis) have shown similar mean score of quality of life ( 2.65 and 2.68, respectively) and inferior to those patients with 3 to 8 points (less severe scoliosis) with mean 3.29 points in the SRS-30 questionnaire. 
The patients with a higher quality of life scores have more stable, less severe scoliosis, probably caused by slow to stationary curve progression. They probably have already to be adapted to the disease and the regular daily and work-life or, in the opposite, maybe are with progressive curves, still not adapted physically and emotionally without directly impacting the quality of life.

A study with 744 patients with idiopathic scoliosis, carried out in the United States, using the SRS-30 quality of life questionnaire, has shown a pre-surgery mean score of 3.9. In contrast, in our study, the pre-surgery score was 3.3. This difference may be related to the worsening of quality-of-life related to the waiting list's time since most patients spend on the list for more than one year waiting for the surgery. In all domain of the SRS-30 questionnaire, our patients' scores were lower than in the US study. The association between the time spent on waiting lists and the decrease in life quality should be better evaluated in future research [16]. Future studies should better evaluate the association between the time spent on waiting lists and the decrease in life quality in future research.

In the analysis grouped by the domains function/activity, pain, self-image, mental health and satisfaction with the treatment, group A (less severe/aggressive scoliosis) has shown better scores in all questions, with a statistical difference in the function/ activity domains $(\mathrm{p}=0.004)$ and self-image $(\mathrm{p}=0.017)$. This data may reflect that the patients with stable scoliosis perform daily-life activities in an adapted way, and they are more self-confident in their physical appearance.

In group B (moderately, severe/aggressive scoliosis) and C (severe/aggressive scoliosis), both have similar results in all domains, with no statistical difference.

The satisfaction domain with the treatment has shown the slightest mean difference between groups, with group A (3.37), B (3.28) and $\mathrm{C}$ (3.32), attributed to the standardisation of the treatment to the patients, regardless of the position on the waiting list, being the item with the highest average score of 3.32 .

Multicentric studies will be necessary to validate and apply these SSS-IoT risk score significantly and comprehensively. Furthermore, future studies will include a control group and individually evaluate the association between the quality-of-life questionnaire domains and each progression factor.

\section{Conclusion}

The quality of life ought to be the primary concern in patients with adolescent idiopathic scoliosis. In our population, the progression of the disease is moderately associated with quality of life. It is possible to gather that the patients who have already suffered from progression are now in a stable course of the disease, well-adapted, and presented a better quality of life than those who are still suffering from progression.

\section{Limitations}

The prolonged waiting time for the surgery in our study is significant. Most of the patients wait longer than one year for the surgery. They are often lately diagnosed in their respective places of origin, many of them entirely after skeletal maturity. This scenario limits the analysis and cannot establish a statistical association between the waiting time and the quality of life.

The geographic dimensions, social-economic and demographic disagreements of our country may cause a delay in the diagnosis. The lack of knowledge of this disease in the laic population often leads to delayed times in seeking specialised health care.

Furthermore, the surgery's complexity and the resources needed to perform the surgery and its associated high direct and indirect costs may explain the extended time waiting. 


\section{Authors' contribution}

Contribution of each author: 1. Substantial contribution to the conception, design of the work, acquisition, analysis and interpretation of data for the work; Writing the work. 2. Substantial contribution to the conception of work, writing of the work and critical review of its intellectual content; 3. Data collection and interpretation. 4. Critical review of the text and intellectual content. 5. Final approval of the version of the manuscript to be published; 


\section{References}

1. Scoliosis Research Society (2020) Adolescent Idiopathic Scoliosis, Brazil.

2. Newton PO, Harms Study Group (2020) Idiopathic scoliosis the Harms Study Group treatment guide, New York: Thieme, USA.

3. Weinstein SL, Ponseti IV (1983) Curve progression in idiopathic scoliosis. J Bone Joint Surg Am 65: 447-55.

4. Buckwalter JA, Yaszay B, Ilgenfritz RM, Bastrom TP, Newton PO (2013) Analysis of Intraoperative Neuromonitoring Events During Spinal Corrective Surgery for Idiopathic Scoliosis. Spine Deform 1: 434-8.

5. Clark S (2008) Waiting times for scoliosis surgery. The Lancet 371: 10-1.

6. Ahn H, Kreder H, Mahomed N, Wright J (2007) Adolescent idiopathic scoliosis waiting time (AWAIT) study: detrimental effects of surgical wait times in a publicly funded health care system.

7. Miyanji F, Newton PO, Samdani AF, Shah SA, Varghese RA, et al. (2015) Impact of Surgical Waiting-List Times on Scoliosis Surgery: The Surgeon's Perspective. Spine 40: 823-8.

8. Avanzi O, Landim, Meves R, Caffaro M, Umeta R (2008) Adolescent idiopathic scoliosis: correlation between radiographic correction parameters and clinical treatment outcome [Escoliose idiopática do adolescente: correlação entre parâmetros radiográficos de correção e resultado clínico de tratamento]. Coluna/Columna 7: 201-8.

9. Carneiro Neto NJ, Umeta R, Meves R, Caffaro MFS, Landim É, et al. (2012) Demographic study of patients with spinal deformities who are awaiting surgery in a tertiary hospital of high complexity. Coluna/Columna 11: 219-22.

10. Mantovani F (2009) Queue for scoliosis surgery is critical in services of the SUS [Fila por cirurgia de escoliose é crítica em serviços do SUS], Brazil.

11. USP Digital Library of Theses and Dissertations (2019) Elaboration and application of a surgical priority stratification scale in patients with adolescent idiopathic scoliosis by Ono AH de A [Elaboração e aplicação de escala de estratificação de prioridade cirúrgica em pacientes portadores de escoliose idiopática do adolescente], Universidade de São Paulo, Brazil.

12. Oliveira L, Marchi L, Coutinho E, Pimenta L (2010) A radiographic assessment of the ability of the extreme lateral interbody fusion procedure to indirectly decompress the neural elements. Spine 35: S331-7.

13. Taylor MC, Hadorn DC (2002) Steering Committee of the Western Canada Waiting List Project. Developing priority criteria for general surgery: results from the Western Canada Waiting List Project. Can J Surg 45: 351-7.

14. Ministério da Saúde (2017) SUS will have a single line in each state for elective surgeries [SUS terá fila única em cada estado para cirurgias eletivas], Brazil.

15. Roussouly P, Labelle H, Rouissi J, Bodin A (2013) Pre-and post-operative sagittal balance in idiopathic scoliosis: a comparison over the ages of two cohorts of 132 adolescents and 52 adults. Eur Spine J 22: 203-15.

16. Roberts DW, Savage JW, Schwartz DG, Carreon LY, Sucato DJ, et al. (2011) Male-Female Differences in Scoliosis Research Society-30 Scores in Adolescent Idiopathic Scoliosis. Spine Phila Pa 36: E53-9. 


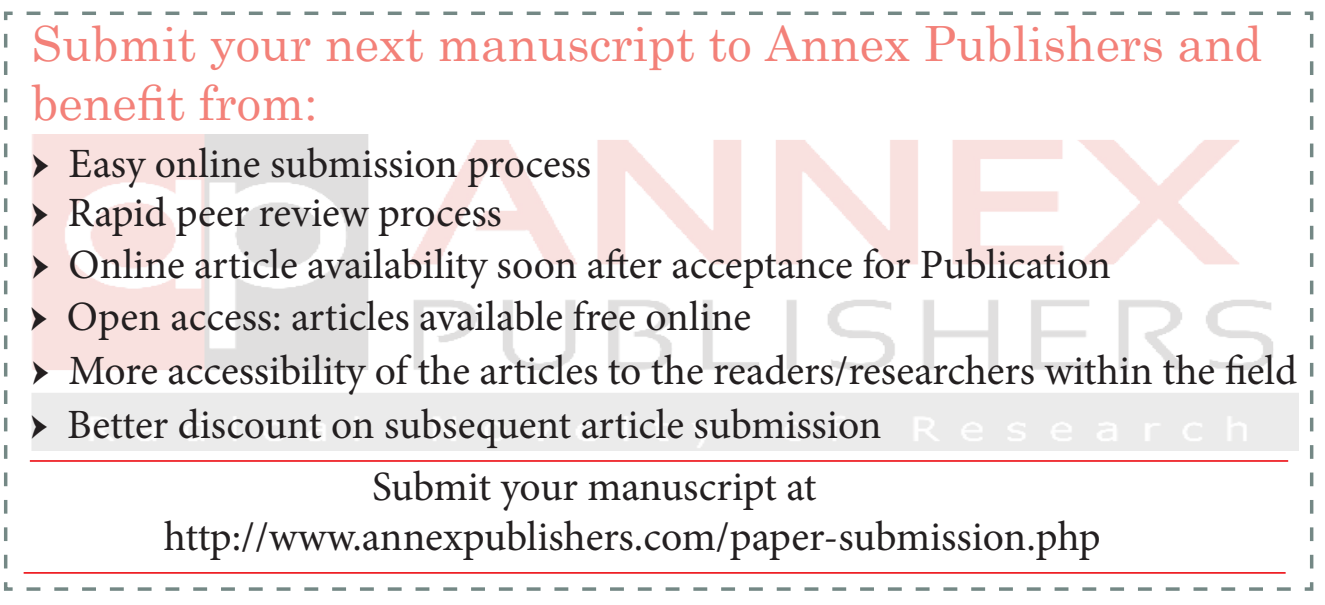

